\title{
Intermittent behavior in the transition region and the low corona of the quiet Sun
}

\author{
S. Patsourakos ${ }^{1,2}$ and J.-C. Vial ${ }^{3}$ \\ 1 US Naval Research Laboratory, Space Science Division, Washington, DC 20375, USA \\ 2 Mullard Space Science Laboratory, University College London, Holmbury St Mary, Dorking, \\ Surrey, RH5 6NT, UK \\ 3 Institut d'Astrophysique Spatiale, Université Paris XI - CNRS, Bât. 121, 91405 Orsay Cedex, France
}

Received 12 June 2001 / Accepted 24 January 2002

\begin{abstract}
We present an analysis of light-curves obtained in the O IV and Ne VIII transition region and low corona lines, that were simultaneously recorded in a quiet Sun region by SUMER/SOHO. By using the flatness spectrum of the observed light-curves we searched for intermittency signatures. It was found that a significant proportion of points in the observed area exhibit clear indications of intermittency, irrespectively of their intrinsic intensity. Our findings give favor to an impulsively heated transition region and corona via intermittent-type MHD turbulence.
\end{abstract}

Key words. Sun: corona - Sun: transition region - Sun: UV radiation

\section{Introduction}

A widely-accepted solution to the coronal heating problem remains still elusive even though considerable theoretical and observational effort has been and is still being made. One of the main-stream schools for coronal heating asserts that the heating has an impulsive nature in time and space and results from a swarm of nanoflares (e.g. Parker 1988). Searching for signatures of coronal heating processes in emissions formed under transition region (TR) and lower corona conditions could be advantageous because the effects of rapid variations originally taking place in the corona can be more easily detected in the TR given its smaller than the corona spatial scales and the relatively small reaction times of TR plasmas to changes of their thermodynamic properties. Hence the TR and the low corona may be quite often viewed as a gauge of the activity taking place in the corona.

A common characteristic of a number of models which build on the idea of a nanoflare-heated corona, is the onset of an intermittent spatio-temporal behavior (e.g., Galsgaard \& Nordlund 1996; Einaudi et al. 1996; Georgoulis et al. 1998; Dmitruk \& Gomez 1999; Galtier 1999). As such, it is very important to search for signatures of intermittency in signals from the TR and low corona, to get insight into coronal and TR heating. We are fortunate to pursue such studies now, since very high quality space-borne observations are currently been available.

Send offprint requests to: S. Patsourakos, e-mail: spatsourakos@buttercup1.nrl. navy.mil
It is indeed the aim of the present paper to search for signatures of intermittency in high temporal and spatial resolution time-series of transition region and lower corona spectral lines intensities. This paper is organized as follows: in Sect. 2 we describe our observations and data while Sect. 3 is dedicated to a description of the tools used to search for intermittency signatures. Finally, in Sect. 4 we discuss our results and give our conclusions.

\section{Observations and data}

Observations were made using the SUMER spectrometer on SOHO (Wilhelm et al. 1995; Wilhelm et al. 1997; Lemaire et al. 1997) on 8 February 1998 in a quiet Sun region around the Sun center. A $1 \times 300 \operatorname{arcsec}^{2}$ entranceslit, with a pixel size of about 1 arcsec along the slit was used, and spectral windows 50 pixels wide (to ensure that the full line plus some continuum were obtained) centered in the O IV lines at 787.7 and $790.2 \AA$ and the Ne VIII lines at 770.4 and $780.3 \AA$ were extracted every $35 \mathrm{~s}$. The solar rotation was compensated every $143 \mathrm{~s}$ by moving the slit to the west with 0.38 arcsec steps. Thus the same region on the Sun was observed for a total duration of 7.6 hours.

We first applied standard corrections to the raw data including flat-fielding, destretching, removal of the local gain depression and correction for non-linearities in the instrument response in order to get rid of a number of instrumental peculiarities (Wilhelm et al. 1997). By integrating over the line profiles (after a background was 

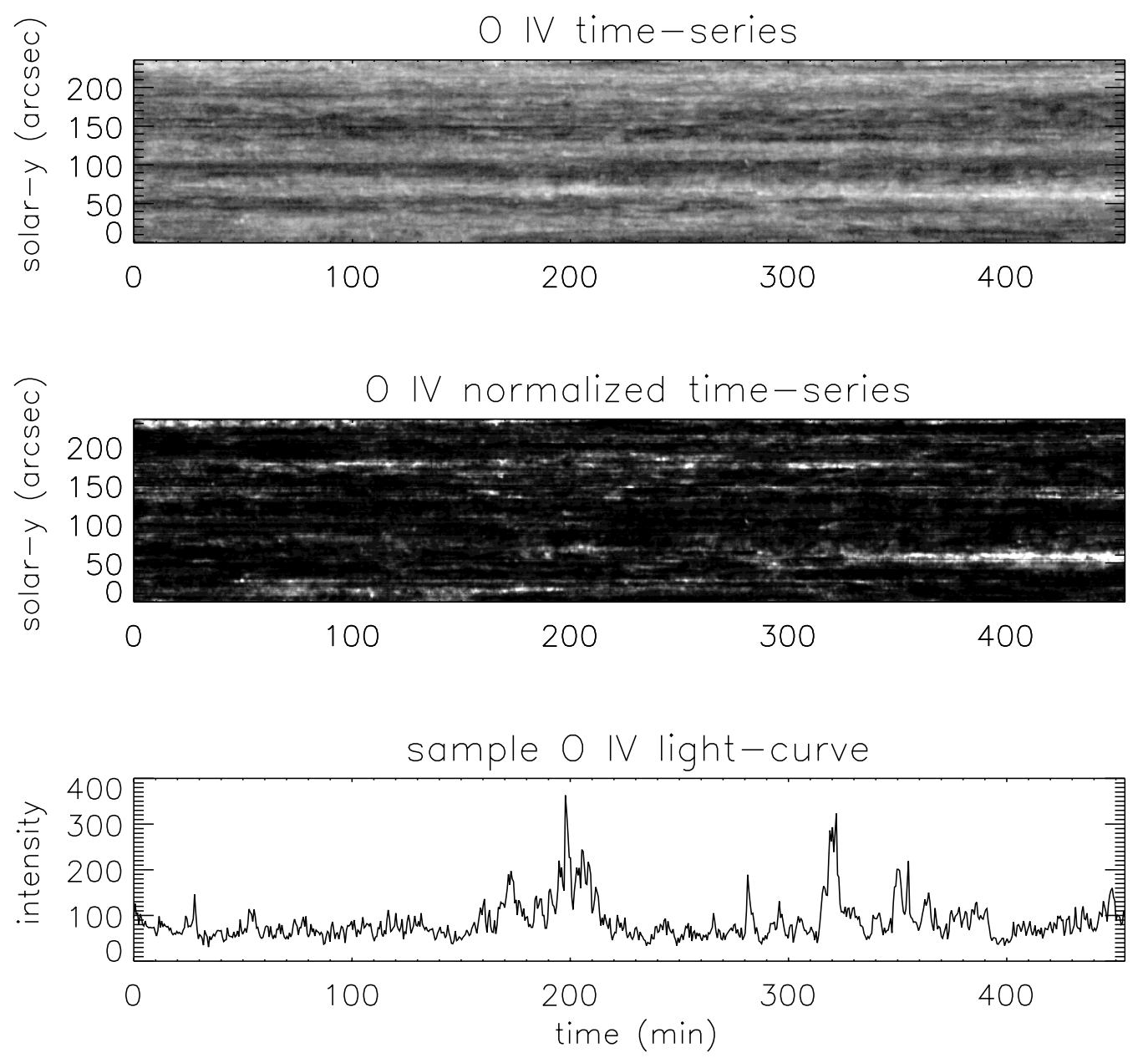

Fig. 1. Time- "solar-y" diagrams for a) the OIV intensity (top panel, logarithmic scaling in intensity) b) the O IV intensity which has been normalized by the minimum value of the light-curve at each spatial pixel (middle panel, linear scaling in intensity) and c) O IV sample light-curve for spatial pixel 100 along the slit (bottom panel).

subtracted) we produced light-curves for each point along the slit. For our analysis we focus on the O IV and Ne VIII lines at 790.2 and $770.4 \AA$ since they are brighter than the other O IV and Ne VIII lines, for ensuring an exploitable signal-to-noise ratio. These two lines offer a good temperature coverage of the transition region and the lower corona since they have formation temperatures of $10^{5.2}$ and $10^{5.8} \mathrm{~K}$ correspondingly. For our analysis we use a 235 pixels long portion of the slit.

An example of the obtained light-curves is given in Fig. 1 for O IV. Along the slit one can observe (Fig. 1, top panel) an alternation of bright and dark portions reminiscent of network and cell elements which roughly speaking preserve their identity during the duration of our observations. We can note the existence of intensity bursts that are unevenly spaced in time. The above remark can be better evidenced in the middle panel of Fig. 1 where we plot the light-curves normalized to their minimum values in order to better visualize such bursts irrespectively to their intrinsic intensity. It can be seen that these bursts seem to occur both in dark and bright regions. Finally, we show (bottom panel of Fig. 1) a sample light-curve at a point selected randomnly alon the slit. We can readily note the existence of spikes in the above time-series all over its duration. The above behavior is indicative of intermittency with switches between bursts and quiescent phases taking place randomly in time.

\section{Measuring the intermittency in a light-curve}

In order to materialize the hints for intermittency we had in the previous section, a more rigorous analysis method is needed. A standard tool to infer for intermittency is the flatness spectrum of the examined signal (Frisch 1995). Following Frisch (1995) we can define the flatness $F(\Omega)$ (fourth order) spectrum of a high-pass filtered version $i_{\Omega}(t)$ of the initial light-curve $i(t)$ corresponding to a temporal scale $\Omega$ as:

$F(\Omega)=\frac{<i_{\Omega}^{4}(t)>}{<i_{\Omega}^{2}(t)>^{2}}$,

where the brackets denote averaging over the duration of the light-curve and $t$ is the time. For building a version of the original light-curves at each spatial scale been selected, we applied a wavelet transform to the original lightcurves (e.g., Farge 1992). Such a transform can be viewed 
as a generalization of the Fourier transform, where a filter (here the wavelet) with an adjustable resolution is applied to the data. Because of their localising properties both in time and temporal scale, wavelet transforms are particulary suitable for analysing signals rapidly varying in time (e.g., Lawrence et al. 1999). We note here that the wavelet filter does not act the same way as a high-pass filter does, since it isolates features at the selected scale rather than selecting all the features that are larger than the selected scale. The wavelet acts as a magnifying lens, enhancing features comparable to its resolution (for our case the selected temporal scale). For our case it is sufficient to use the simplest wavelet: the Haar. For a given temporal scale $\Omega$ the Haar wavelet is defined as a filter function which equals to -1 in the interval $-\frac{\Omega}{2} \leq t<0,1$ in the interval $0 \leq t \leq \frac{\Omega}{2}$ and 0 otherwise. In the following we should keep in mind that a Gaussian signal has a constant with the temporal scale flatness spectrum, with a value of 3 (Frisch 1995). On the other hand an intermittent signal is characterized by a flatness spectrum with values larger than the Gaussian value.

The flatness spectrum analysis, as a probe of intermittent behavior both in space and time, has been extensively used in a great number of problems including turbulent flows (e.g., Frisch 1995 and references within), solar sunspot number (Lawrence et al. 1995), photospheric flow fields (Lawrence et al. 1999) magnetic and kinetic energy as well as the dissipation rate of modeled coronal loops (Dmitruk et al. 1998), and solar wind magnetic field and velocity fluctuations (Bruno et al. 1999).

\section{Results and discussion}

The temporal flatness spectrum for the OIV line is presented in Fig. 2 (top panel). It can be seen that a very significant fraction of the observed region, for temporal scales from $35 \mathrm{~min}$ down to around $1 \mathrm{~min}$ (the smallest temporal scale we can access because of the temporal resolution of our observations), has larger than 3 value of the corresponding flatness spectrum and thus they are in an intermittent state. Moreover almost the whole observed region exhibits is an intermittent state at temporal scales smaller than $5 \mathrm{~min}$. The flatness spectrum does not consist of points randomly scattered in the spatial position - temporal scale space; rather they are organized as vertical (to the spatial position) spikes. These spikes typically have a width of $\approx 5$ arcsec and are characterized by a decrease of the value of the flatness spectrum with the temporal scale. The latter is a typical property of intermittent activity which is more pronounced at small temporal scales. We can finally note that those spikes do not preferentially lie in a particular kind of spatial structure (see the upper and the bottom panel of Fig. 2); they can be found either in bright or dark portions along the slit which could correspond to network and cell. Thus we may conclude that the intermittency is a common characteristic of the different structures found in the transition region, irrespectively of their intensity. We found basically the same
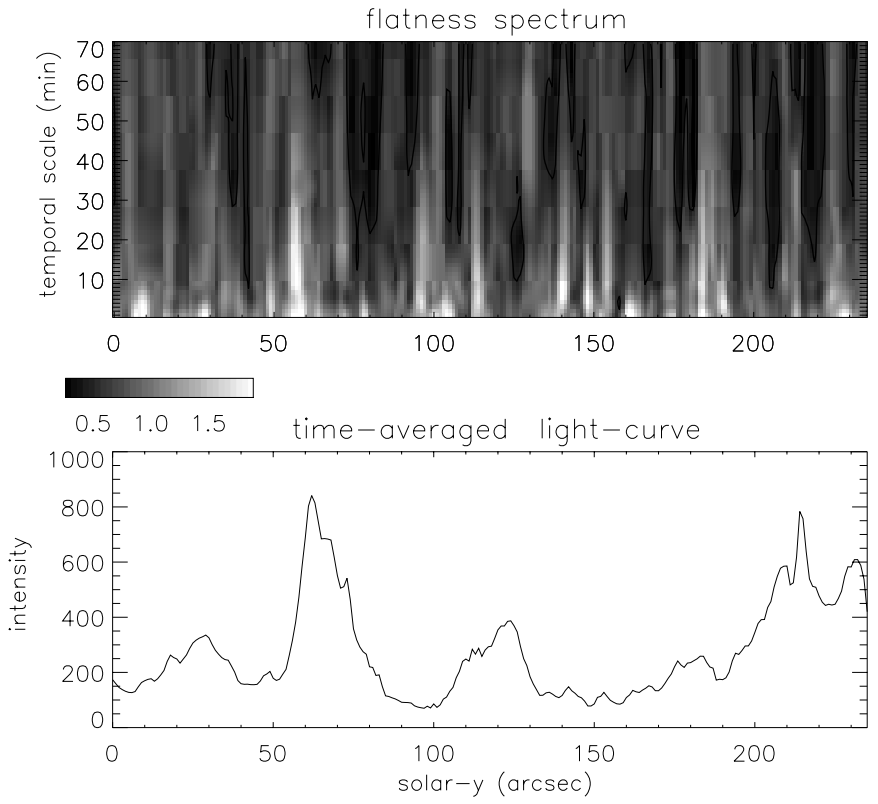

Fig. 2. Temporal flatness spectrum of the OIV line intensity along the slit (top panel-logarithmic scaling). The horizontal color-bar corresponds to the logarithm of the flatness. The overplotted contours indicate points with flatness below 3 . Time-averaged O IV intensity along the slit (bottom panel).

behavior in the Ne VIII line. However in the Ne VIII flatness spectrum the spikes are somehow less and do not always coincide with their equivalents in O IV. This can be explained by a "localization", in a very restricted temperature band, of the processes behind the intermittent activity. Another possibility can be the smaller radiative times in O IV than in Ne VIII. In order to better visualize the behavior of the intermittency throughout the totality of the observed field, we present in Fig. 3 the spatiallyaveraged temporal flatness spectra for O IV and Ne VIII. In both lines the flatness increases with decreasing temporal scale, although we can witness (as already been made above), that in Ne VIII the intermittence is significantly not as strong as in OIV. Let us note here that we found that Fourier high-pass filtering and other types of wavelets (Mexican Hat and Morlet; see Torrence \& Compo 1998) also provide a decreasing flatness with temporal scale. By a series of Monte-Carlo simulations we found that instrumental effects such as flat-field and photon counting noise as well as pointing jitter do not have a significant bearing on the derived flatness spectra (we found a mean difference of 0.003 between the simulated and the derived from the observations flatness spectra).

The main result of this work is that intermittent activity is quite ubiquitous throughout the TR of the quiet Sun and in fact increases when the time scales under consideration get smaller and smaller. This lends considerable support to a series of theoretic models of coronal heating (see the references in the introduction) which find their route to the Parker's nanoflare heated corona via MHD turbulence. All these models lead to an intermittent behavior in the time-variation of a number of physical parameters 
Spatially-averaged flatness

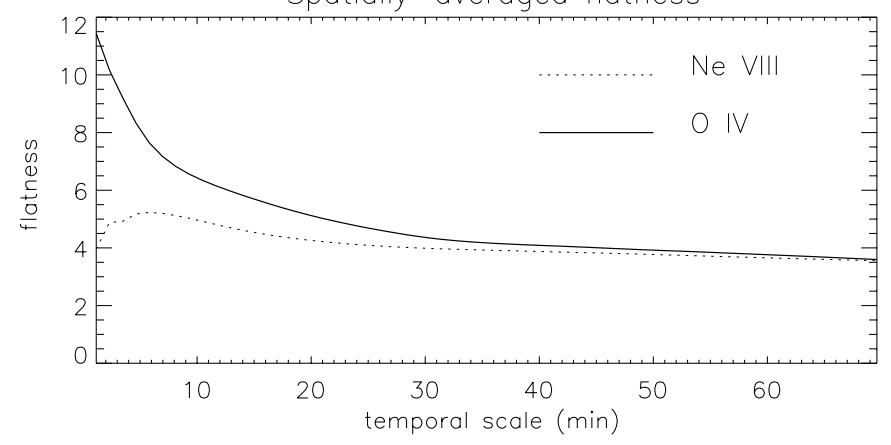

Fig. 3. Spatially averaged temporal flatness spectra for O IV (continuous line) and for Ne VIII (dashed line).

including the dissipated energy. The radiative losses are related to the dissipated energy since one part of it is lost as radiation. Thus, we should expect a correlation between the observed line intensities and the dissipated energy and consequently intermittent bursts in the observed light-curves, even though a smoothing may be expected in the observed light-curves due to the limited (and not infinitely small) time it takes a plasma to react to modifications of its thermodynamic properties by radiation. Such bursts could be related to well-known types of transient events such as explosive events (e.g., Dere et al. 1987; Innes et al. 1997; Patsourakos \& Vial 2001), microflares (e.g., Porter et al. 1987), blinkers (e.g., Harrison 1997) etc., with however the above events been presumably just the "tip of the iceberg" of a whole hierarchy of events (e.g., Parker 1988) that are currently non-resolved by our instrumentation, given also the fact that such events are not too numerous to account for the almost omni-present observed intermittency. Another point which deserves some comment here is the fact that the models above are originally intended to model large scale coronal loops. However we can assert that they could be applied also to the quiet Sun, via the interaction of small loops in the frame of the "magnetic carpet" concept (Schrijver et al. 1997) for example (see also the relevant discussion in Aletti et al. 2000). It is just the macroscopic spatial scales of the structures involved which are different: the underlying physical processes could be the same. It is very tempting to draw a link between these small loops and the spikes observed in the flatness spectrum. What it is really needed from MHD turbulence models is that they could be able to cope with the plasma local thermodynamics, in order to reproduce the spectral emission of the plasma which provides a common ground for comparisons between observations and models. We note that most of MHD turbulence simulations assume incompressibility which by definition does not cater for variations of local thermodynamic parameters (i.e., density and temperature) which are essential elements for determining the radiative signatures of the plasma. Some first steps trying to reconcile this deficiency, have been taken for a number of simplistic cases by Walsh \& Galtier (2000). Very interestingly these authors found, that for some of the cases they considered, bursts appear in both the plasma temperature and density as it was anticipated above. These bursts result from intermittent energy release followed by its dissipation. To note here that the bursts in the dissipated energy are by far more stronger in magnitude than the bursts in density and temperature, which may make feasible the observational detection only for the stronger among them.

Our results are broadly consistent with the work of Aletti et al. (2000). These authors found that the intensity distributions of the quiet Sun coronal snapshot images they analyzed, show departures from Gaussianity at large intensities even with a degraded spatial resolution. They interpreted the above findings as suggestive of a forced turbulent system characterized by an intermittent behavior, having dissipation scales much more smaller than the spatial resolution. The relevance of an MHD turbulence regime in the quiet Sun has also been demonstrated in two recent articles by Berghmans et al. (1998) and by Chae et al. (1998) by totally independent means than ours. They analyzed respectively temporal/spatial intensity power spectra and non-thermal velocities in the quiet Sun and found that they are consistent with predictions from MHD turbulence theory. As a matter of fact, our findings in favor of intermittent MHD turbulence could have a significant bearing on the way that observational data are investigated for scaling properties (e.g., exponents) of MHD turbulence. Higher order than 2 (power spectrum) moments may need to be considered.

What is clearly needed for the future is on the one hand a more realistic coupling between heating and the hydrodynamics and on the other hand an extension of our study to other structures (e.g. active regions) and to bi-dimensional spatial fields seeking for spatio-temporal intermittency in 2-D.

Acknowledgements. S. Patsourakos acknowledges postdoctoral support by the PPARC. The research of S. Patsourakos was supported in part by NASA and ONR. We would like to thank the referee, Dr. L. Milano for his comments/suggestions that helped to improve this paper. The authors wish to thank S. Galtier, M. Georgoulis, U. Frisch, P. Lemaire, A. Mangeney and M. Velli for very useful and enlightening discussions during the course of this work. The SUMER project is financially supported by DLR, CNES, NASA and the ESA Prodex programme (Swiss contribution). SOHO is a mission of international cooperation between ESA and NASA. Wavelet software was provided by C. Torrence and G. Compo, and is available at URL: http://paos. colorado.edu/research/wavelets.

\section{References}

Aletti, V., Velli, M., Bocchialini, K., et al. 2000, ApJ, 544, 550 Berghmans, D., Clette, F., \& Moses, D. 1998, A\&A, 336, 1039 Bruno, R., Bavassano, B., Pietropaolo, E., et al. 1999, GRL, 26, 3185

Chae, J., Schühle, U., \& Lemaire, P. 1998, ApJ, 505, 957

Dere, K. P., Bartoe, J.-D. F., Brueckner, G. E., et al. 1987, Sol. Phys., 114, 223 
Dmitruk, P., Gomez, D., \& DeLuca, E. E. 1998, ApJ, 505, 974

Farge, M. 1992, Ann. Rev. Fluid Mech., 24, 395

Frisch, U. 1995, Turbulence (Cambridge University Press)

Galsgaard, K., \& Nordlund, A. 1996, J. Geophys. Res., 101, 13445

Galtier, S. 1999, ApJ, 521, 483

Georgoulis, M. K., Velli, M., \& Einaudi, G. 1998, ApJ, 497, 957

Einaudi, G., Velli, M., Politano, H., \& Pouquet, A. 1996, ApJ, 457, L113

Harrison, R. A. 1997, Sol. Phys., 175, 467

Innes, D. E., Inhester, B., Axford, W. I., \& Wilhelm, K. 1997, Nature, 386,811

Lawrence, J. K., Cadavid, A. C., \& Ruzmaikin, A. A. 1995, ApJ, 455, 366
Lawrence, J. K., Cadavid, A. C., \& Ruzmaikin, A. 1999, ApJ, 513,506

Lemaire, P., Wilhelm, K., Curdt, W., et al. 1997, Sol. Phys., 170,105

Parker, E. N. 1988, ApJ, 330, 474

Patsourakos, S., \& Vial, J.-C. 2001, Sol. Phys., 203, 39

Porter, J. G., Moore, R. L., Reichmann, E. J., et al. 1987, ApJ, 323,380

Schrijver, C. J., Title, A. M., Van Ballegooijen, A. A., et al. 1997, ApJ, 487, 424

Walsh, R. W., \& Galtier, S. 2000, Sol. Phys., 197, 57

Wilhelm, K., Curdt, W., Marsch, E., et al. 1995, Sol. Phys., 162,189

Wilhelm, K., Lemaire, P., Curdt, W., et al. 1997, Sol. Phys., 170,75 